Case Report

\title{
CO-INFECTION OF SARCOCYSTIS SP. AND HADJELIA TRUNCATA IN FANTAIL PIGEONS (COLUMBA LIVIA DOMESTICA)
}

\author{
M. KHORDADMEHR ${ }^{1}$, V. R. RANJBAR ${ }^{2}$, P. SHAHBAZI $^{1} \&$ M. ZEINODDIN ${ }^{1}$ \\ ${ }^{1}$ Department of Pathobiology, Faculty of Veterinary Medicine, University of \\ Tabriz, Iran; ${ }^{2}$ Veterinary Clinic of Avian Diseases, Yazd Province, Yazd, Iran
}

\section{Summary}

Khordadmehr, M., V. R. Ranjbar, P. Shahbazi \& M. Zeinoddin, 2018. Co-infection of Sarcocystis sp. and Hadjelia truncata in fantail pigeons (Columba livia domestica). Bulg. J. Vet. Med., 21, No 1, 115-121.

\begin{abstract}
Hadjelia truncata belongs to the family Habronematidae which affects different groups of birds such as Columbiformes. A large number of Sarcocystis sp. may infect birds as intermediate hosts, but wild Columbiformes, include pigeons, are rarely affected. The present study describes mixed infection of two pigeon flocks with sarcocystosis and nematodiasis (H. truncata) which had neurologic and gastrointestinal clinical signs. The common clinical signs included progressive weight loss, pectoral muscle atrophy, white diarrhoea, depression, torticollis, paralysis, trembling, and $23.4 \%$ mortality. At necropsy, a large number of nematodes were detected in the gizzards and diagnosed as $H$. truncata in parasitological studies. For greater certainty, histopathological examination was conducted routinely. Different development stage of this nematode associated with severe inflammatory cells infiltration and necrosis were observed in tissue sections. Accidentally, the large number of Sarcocystis cysts was observed in tunica muscularis mucosa of gizzard associated with infiltration of inflammatory cells, hyaline degeneration and necrosis around degenerated cysts.
\end{abstract}

Key words: gizzard, Hadjelia truncata, inflammatory cells, pigeon, sarcocystosis

Pigeons are encountered in all regions of the world except for the poles. They live side by side with humans and other animal species in the nature and they are bred as a source of food as a hobby, symbol and for experimental aims (Harlin, 1994). Pigeons have a role in spreading some zoonoses to people and are a reservoir of many parasitic diseases for poultry ( $\mathrm{Pi}-$ asecki, 2006).
Hadjelia truncata belonging to the family Habronematidae and order Spirurida (Anderson, 2000), affects different groups of birds such as Coraciiformes, Columbiformes and rarely Galliformes (Junker \& Boomker, 2007). Clinical symptoms such as weight loss, diarrhoea, weakness and death were observed in infected pigeons (Geyhan, 2007; Appleby et al., 1995). The adult parasite resides in the gizzard of the bird and the eggs are 
present in the faeces. Various kinds of beetles, mainly Alphitobius diaperinus, have been identified as intermediate hosts (Junker \& Boomker, 2007). There are several reports of pigeon infestation with this parasite from Egypt (Tadros \& Iskander, 1997), Iraq (Al-Attar \& Abdul-Aziz, 1985), Cyprus (Appleby et al., 1995) and Iran (Razmi et al., 2007; Radfar et al., 2011; Nabavi et al., 2013).

A large number of Sarcocystis spp. (Protozoan; Apicomplexa) may infect birds as intermediate hosts, but wild Columbiformes, which include pigeons, are rarely affected (Ecco et al., 2008; Olias et al., 2009). Among the few species affecting domestic poultry are $S$. horvathi and $S$. wenzeli, which infect chickens, and $S$. rileyi, for which ducks are intermediate hosts (Riley, 1931; Wenzel et al., 1982). S. falcatula has been known to cause clinical disease in pigeons only after experimental infection; whether this species is pathogenic under natural conditions is not known (Box \& Smith, 1982). In a previous study, $S$. falcatula-like infection have caused death in three free-roaming Victoria crowned pigeons (Goura victoria) in the USA (Suedmeyer et al., 2001). An early literature report described an emerging neurologic disease with lethal outcome for domestic pigeons (Columba livia f. domestica) in Berlin, Germany, caused by a novel Sarcocystis sp. (Olias et $a l ., 2009$ ). Moreover, newly characterised non-pathogenic species $S$. columbae have been found in wood pigeons (Columba palumbus) in Germany (Olias et al., 2010b). Clinical signs in naturally infected pigeons, similar to those caused by Paramyxovirus-1 or Salmonella Typhimurium infection, were depression, polyuria, torticollis, opisthotonus, paralysis, trembling, and death. Pigeons had nume- rous parasitic cysts in their muscles (Olias et al., 2010a).

The present study describes (for the first time) mixed infection of two pigeon flocks (with 94 pigeons) with sarcocystosis and nematodiasis (Hadjelia truncata) which had neurologic and gastrointestinal clinical signs.

The owner of two pigeon (Columbia livia) flocks with 94 birds (in Yazd Province; central part of Iran) complained from gastrointestinal and neurological disease manifested by progressive weight loss specially with pectoral muscle atrophy, white diarrhoea, vomiting, weakness, depression, torticollis, paralysis, trembling, and 23.4\% (22 cases) mortality which occurred 4-6 days after clinical signs. Blind antibiotic therapies were administered to treat the sick birds, but no advantage was observed.

Six new dead bird carcasses were chosen for postmortem inspection. According to the clinical signs, the neurotropic velogenic form of Newcastle disease was suspected at first. For this reason, the viscera and trachea were removed and opened for macroscopic examination. In all cases, the gizzard was larger and softer than normal and distortion was observed. A large number of nematodes was detected beneath the lining of the affected gizzards (Fig. 1). The other organs were macroscopically normal. According to the necropsy, the treatment was performed by levamisole hydrochloride and anhydrous niclosamide which did not appear to be an effective treatment for the nematode.

The worms were collected from the lining of the gizzards and washed by shaking in $0.9 \%$ saline and kept in $70 \%$ ethanol $+5 \%$ glycerin. A number of worms were counted and cleared in lactophenol for identification using a light microscope (Soulsby, 1982). Also, for pathological 
examination, tissue blocks from various parts of the gizzards were processed by conventional methods for preparation of paraffin wax sections. The sections were stained with haematoxylin and eosin and studied with light microscope.

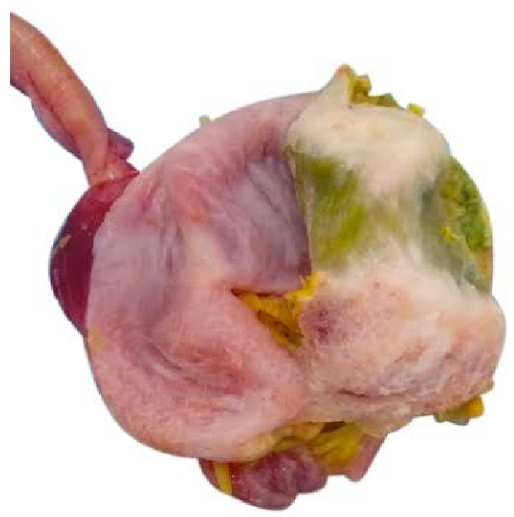

Fig. 1. Macroscopic appearance of the large number of nematodes beneath the lining of the affected gizzards.

A large number of nematodes were observed under the koilin layer of the gizzard during necropsy. The mean worm burden was 115 worms per case (30 male and 85 female). The morphology of nematodes was examined by light microscope. The length of male and female were 7-11 $\mathrm{mm}$ and $15-20 \mathrm{~mm}$, respectively. Microscopical examination of cephalic region revealed two lateral lips with winged appearance and a cylindrical pharynx (Fig. 2A). Moreover, in the caudal region (Fig. 2B, C), two obvious unequal and dissimilar spicules were observed in the male nematode. The tail was coiled and two wide caudal alae and papillae were present. Pathological study of tissue samples was also done. Different developmental stages of this nematode (egg with length $43-45 \mu \mathrm{m}$ and width of $20-30 \mu \mathrm{m}$, larva and adult) were observed in tissue sections (Fig. 3A, B). The parasite was more prevalent between tunica submucosa and tunica muscularis mucosa of gizzard (Fig. 3C). Severe different inflammatory cells infiltration (including heterophils, eosinophils and lymphocytes) (Fig. 3D) with necrosis was seen in mucosa and submucosa of gizzard.

Accidentally, at pathological study, we observed a large number of microscopic Sarcocystis cysts in the tunica muscularis mucosa of gizzard (Fig. 4A). In non-degenerated cysts (Fig. 4B), the cyst wall seemed smooth or slightly wavy by light microscope. Moreover, a heterophil-rich inlammatory cell population associated with hyaline degeneration and necrosis were observed around degenerated cysts (Fig. 4C, D).

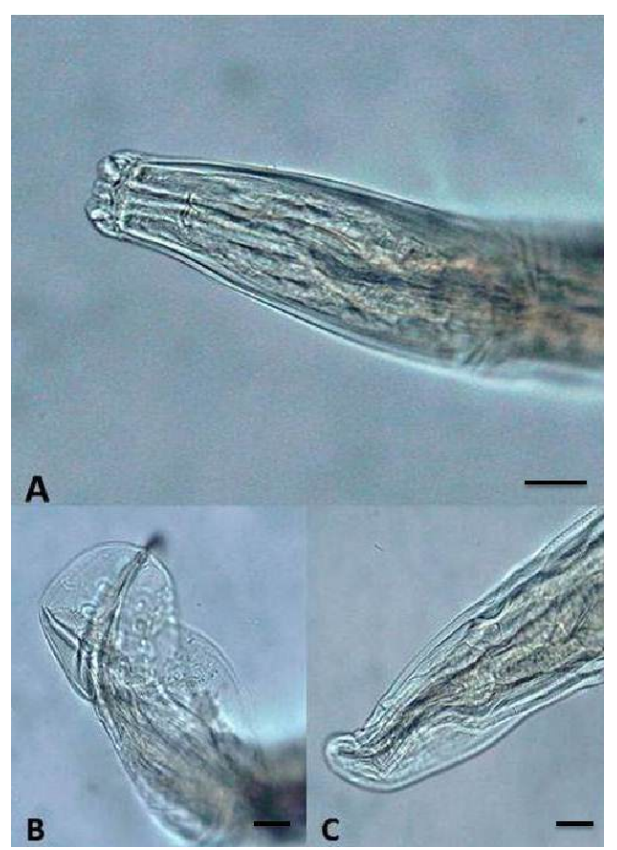

Fig. 2. Anterior and posterior end of Hadjelia truncata. In cephalic region, 2 lateral lips with winged appearance were seen (A). In tail region, two obvious unequal spicules with coiled tail are presented in male (B) but not in the tail of female $(\mathrm{C})(\mathrm{bar}=10 \mu \mathrm{m})$. 


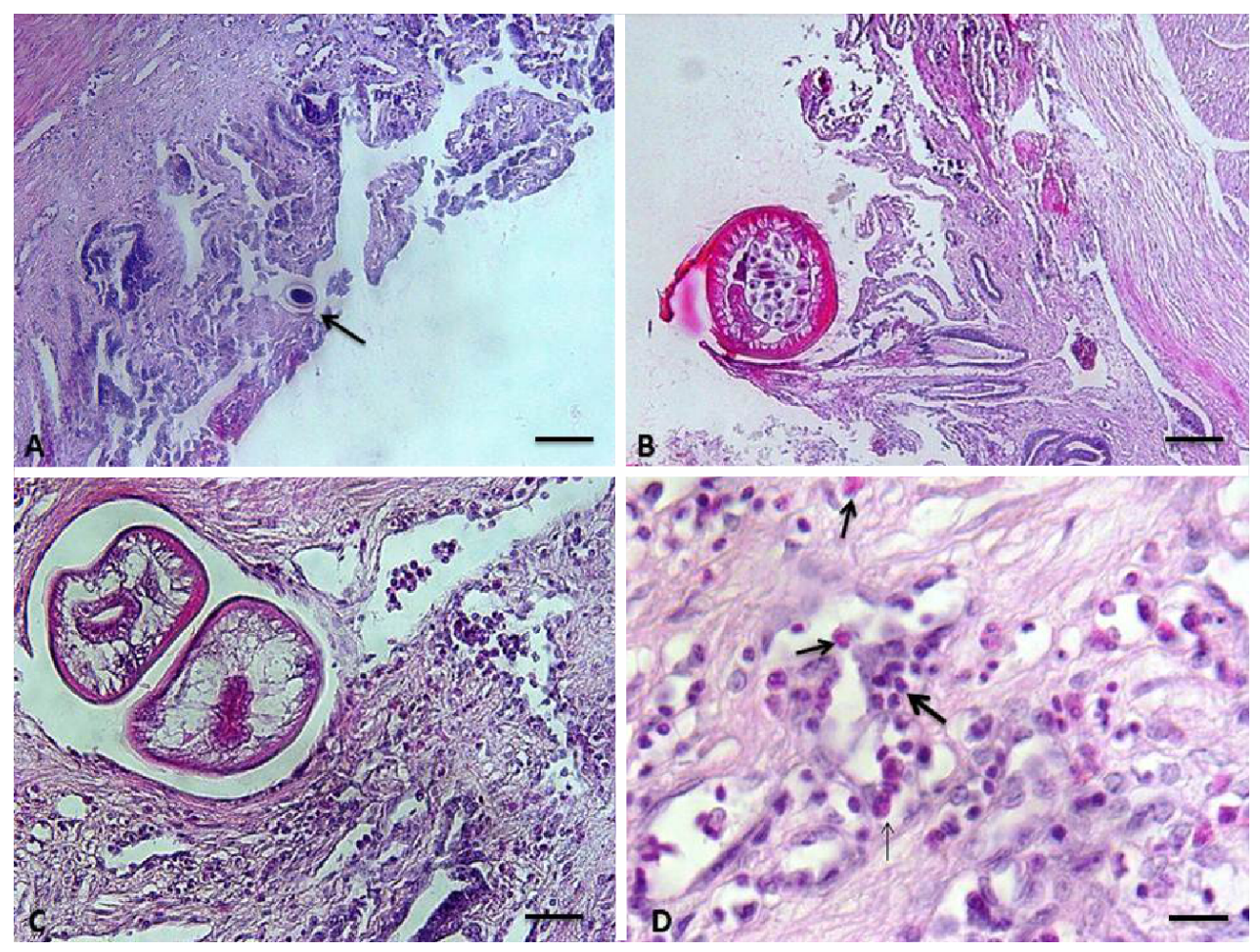

Fig. 3. Gizzard, pigeon, nematodiasis. Different developmental stage of this nematode. A) Egg with length $43-45 \mu \mathrm{m}$ and width of $20-30 \mu \mathrm{m}$; arrow (H\&E, bar=300 $\mu \mathrm{m}$ ). B) Adult was observed in tissue sections $(\mathrm{H} \& \mathrm{E}$, bar $=300 \mu \mathrm{m})$. C) The parasite was predominant between tunica submucosa and tunica muscularis mucosa $(\mathrm{H} \& \mathrm{E}$, bar $=200 \mu \mathrm{m})$. D) Inflammatory cells infiltration: heterophil (thin arrow); eosinophil (semithin arrows); lymphocyte (thick arrow) in gizzard mucosa and submu$\operatorname{cosa}(\mathrm{H} \& \mathrm{E}$, bar $=60 \mu \mathrm{m})$.

Both sarcocystosis and nematodiasis (due to Hadjelia truncata) are lethal in pigeons (Olias et al., 2010a; Nabavi et al., 2013). Sarcocystis sp. and Hadjelia truncata in pigeons cause neurological and gastrointestinal symptoms which were seen in the pigeons simultaneously in this study.

In the present study, the nematodes in the gizzard, were identified as Hadjelia truncata. Recently, this parasite has been reported from countries such as Cyprus (Appleby et al., 1995) and Iran (Razmi et al., 2007; Radfar et al., 2011; Nabavi et al., 2013). The most common clinical signs reported by these researchers were nonspecific gastrointestinal symptoms and included body weight loss, poor feed consumption, diarrhoea, weakness and increased mortality. Also, diagnosis is based on the necropsy and observation of the nematodes under the koilin layer of the gizzard. Nabavi et al. (2013) reported severe clinical signs and $9.58 \%$ mortality rate in three pigeon flocks containing 637 birds. In another study from Iran however, slight infection $(1.096 \%)$ by this parasite has been reported in a survey of parasites 

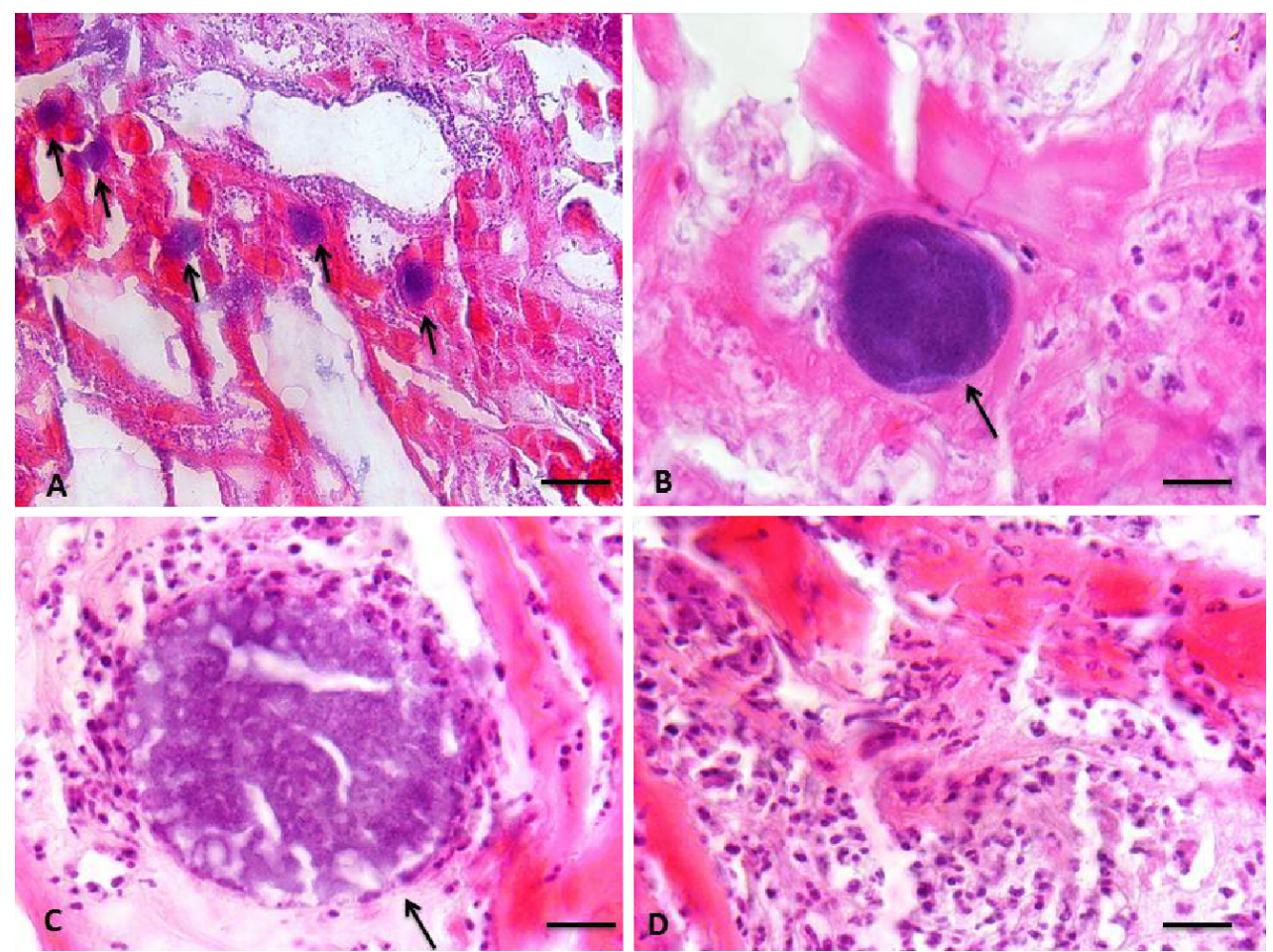

Fig. 4. Gizzard, pigeon, sarcocystosis. A) Large number of microscopic Sarcocystis cysts in tunica muscularis mucosa of gizzard $(\mathrm{H} \& \mathrm{E}, \mathrm{bar}=200 \mu \mathrm{m})$. B) In non-degenerated cysts $(\mathrm{H} \& \mathrm{E}, \mathrm{bar}=60$ $\mu \mathrm{m})$, the cyst wall seemed smooth or slightly wavy. C) Heterophil-rich inflammatory cell population around degenerated cysts $(\mathrm{H} \& \mathrm{E}, \mathrm{bar}=60 \mu \mathrm{m})$. D) Hyaline degeneration and necrosis associated with inflammatory cells around degenerated cysts $(\mathrm{H} \& \mathrm{E}, \mathrm{bar}=60 \mu \mathrm{m})$.

of domestic pigeons (Radfar et al., 2011). It seems that the severity of symptoms seen in affected pigeons of the present study, are more similar to the findings of Nabavi et al. (2013). The cephalic and caudal morphology of Hadjelia truncata nematode was described by Appleby et al. (1995). Moreover, parasitological investigation of the present study, similar to that of Nabavi et al. (2013), showed that the collected nematodes were longer than those described by Razmi et al. (2007). Probably, this resemblance could be because of the similar weather conditions of these two areas in Iran (Sistan and Yazd Provinces).

At histopathological examination, the nematodes were seen in all parts of the gizzard; mucosal surface, serosal surface, junction of tunica submucosa and tunica muscularis mucosa and even between of muscle fibres of muscularis mucosa. There was an inflammatory response in all places where the nematodes were present. Gross and histopathological findings were similar to those reported by other reserchers (Razmi et al., 2007).

Sarcocystis calchasi is the causative agent of pigeon protozoal encephalitis 
(PPE), an emerging neurological disease of the domestic pigeon (Columba livia $f$. domestica) (Olias et al., 2009; Wünschmann et al., 2011). To date, little research has been done in this area. Recently, some researchers reported an emerging neurologic disease with lethal outcome for domestic pigeons (Columba livia f. domestica) in Berlin, Germany, caused by a novel Sarcocystis sp. (Olias et al., 2009). Also, in an experimental study, pigeons infected with high doses of Sarcocystis $s p$. died 7-12 days after infection and showed multifocal severe necrosis with numerous parasitic stages while those infected with lower doses had central nervous signs, which did not develop until 8 weeks after infection and at pathological examination, exhibited marked encephalitis, myositis, and Sarcocystis cysts in skeletal muscles (pectoral, gastrocnemius and neck) but not in the brain. The late occurrence of brain lesions and the absence of parasitic stages in the brain suggested an indirect and currently unknown mechanism of encephalitis (Olias et al., 2010a). The results of another recent study suggested an immune evasion strategy of $S$. calchasi during the early phase and a delayed-type hypersensitivity reaction as cause of the extensive cerebral lesions during the late neurological phase of disease. In this study, according to unsuccessful antibiotic therapy, probably due to the use of levamisole and its effect on the nematodes, gastrointestinal symptoms improved, but neurological symptoms still continued and the pigeons with neurological signs died after 2-3 weeks. In the present study, Sarcocytis cysts were found in the gizzard accidentally, therefore only the gizzard showed a large number of cysts with severe inflamatory cell response in tunica muscularis mucosa. The results of this part suggest that the muscles of the gizzard could be a convenient place for future research of Sarcocystis sp. in the pigeons which has not been reported before.

The present study described a mixed infection of pigeon with sarcocystosis and nematodiasis (Hadjelia truncata) both of which lethal for pigeons, with neurologic and gastrointestinal clinical signs. Because of the severe disorders caused by these two parasites and little knowledge about them in pigeons, especially on Sarcocystis sp., more research in these fields is needed.

\section{ACKNOWLEDGEMENTS}

The authors are grateful to the Faculty of Veterinary Medicine, University of Tabriz, Tabriz, Iran for the financial support.

\section{REFERENCES}

Al-Attar, M. A. \& T. A. Abdul-Aziz, 1985. Hadjelia truncata in pigeons. Veterinary Record, 117, 535.

Anderson, R. C., 2000. Nematode parasites of vertebrates: Their development and transmission. $2^{\text {nd }}$ edn, ABI Publishing, Wallingford, UK., pp. 432-433.

Appleby, E. C., S. L. M. Gibbons \& K. Georiou, 1995. Distortion of the gizzard in Cyprus pigeons (Columba livia) associated with Hadjelia truncata infestation. The Veterinary Record, 136, 561-564.

Box, E. D. \& J. H. Smith, 1982. The intermediate host spectrum in a Sarcocystis species of birds. Journal of Parasitology, 68, 668-673.

Ecco R, M. M. Luppi, M. C. Malta, M. R. Araujo, R. M. Guedes \& H. L. Shivaprasad, 2008. An outbreak of sarcocystosis in psittacines and a pigeon in a zoological collection in Brazil. Avian Diseases, 52, 706-710. 
Harlin, R. W., 1994. Pigeons. The Veterinary Clinics of North America: Small Animal Practice, 24, 157-173.

Junker, K. \& J. Boomker, 2007. Helminths of guineafowls in Limpopo province, South Africa. The Onderstepoort Journal of Veterinary Research, 74, 265-280.

Nabavi, R., J. Khedri \& M. Jahantigh, 2013. High clinical disturbance and mortality in pigeon flocks caused by Hadjelia truncata infection in Sistan, Iran. Turkish Journal of Veterinary and Animal Sciences, 37, 355-357.

Olias, P. A. D. Gruber, A. O. Heydorn, A. Kohls, H. Mehlhorn \& H. M. Hafez, 2009. A novel Sarcocystis-associated encephalitis and myositis in racing pigeons. Avian Pathology, 38, 121-128.

Olias P., A. D. Gruber, A. Kohls, H. M. Hafez, A. O. Heydorn, H. Mehlhorn, M. Lierz, 2010a. Sarcocystis species lethal for domestic pigeons. Emerging Infectious Diseases, 16, 497-499.

Olias P., L. Olias, M. Lierz, H. Mehlhorn, A. D. Gruber, 2010b. Sarcocystis calchasi is distinct to Sarcocystis columbae sp. nov. from the wood pigeon (Columba palumbus) and Sarcocystis sp. from the sparrowhawk (Accipiter nisus). Veterinary Parasitology, 171, 7-14.

Piasecki, T., 2006. Evaluation of urban pigeons (Columbia livia furbana) health status in relatin to their threat to humans health. Medycyna Weterynaryjna, 62, 531535.

Radfar, M. H., S. Fathi, E. Norouzi, M. Mirzaii, \& H. Rezaei, 2011. A survey of parasites of domestic pigeons (Columba liva domestica) in South Khorasan, Iran. Veterinary Research, 4, 18-23.

Razmi, G. R., G. A. Kalidari \& M. Maleki, 2007. First report of the Hadjelia truncata infestation in pigeons of Iran. Iranian Journal of Veterinary Research University of Shiraz, 19, 175-177.

Riley, W. A., 1931. Sarcosporidiosis in ducks Parasitology, 23, 282-285.
Soulsby, E. J. L., 1982. Helminths, Arthropods and Protozoa of Domesticated Animals. $7^{\text {th }}$ edn, Bailliere Tindall, London, pp. 763766.

Suedmeyer W. K., A. J. Bermudez, B. C. Barr \& A. E. Marsh, 2001. Acute pulmonary Sarcocystis-falcatulalike infection in three Victoria crowned pigeons (Goura victoria) housed indoors. Journal of Zoo and Wildlife Medicine, 32, 252-256.

Tadros, G. \& A. R. Iskander, 1975. Hadjelia truncata, a new parasite of pigeons in Egypt and its pathogenicity. Journal of the Egyptian Veterinary Medicine Association, 35, 283-301.

Wenzel, R., M. Erber, J. Boch \& H. P. Schellner, 1982. Sarcocystis infections in domestic fowl in pheasant and in coots Berliner und Münchener Tierärztliche Wochenschrift, 95, 188-193.

Wünschmann, A., A. G. Armien, L. Reed, A. D. Gruber \& P. Olias, 2011. Sarcocystis calchasi-associated neurologic disease in a domestic pigeon in North America. Transboundary and Emerging Diseases, 58, 526-530.

Paper received 11.01.2016; accepted for publication 26.02.2016

\section{Correspondence:}

Dr. M. Khordadmehr $\mathrm{Ph}$. D. of Veterinary Pathology Department of Pathobiology Faculty of Veterinary Medicine University of Tabriz, Tabriz, Iran P.O. Box: 71345-1731

Tel: +98-4136378743

Fax: +98-4136378744

Email: khordadmehr@tabrizu.ac.ir; m.khordadmehr20@gmail.com 